\title{
Direct Comparison of Catalyst-Free and Catalyst-Induced GaN Nanowires
}

\author{
Caroline Chèze ${ }^{1, \dagger}(\varangle)$, Lutz Geelhaar ${ }^{1, \dagger}$, Oliver Brandt ${ }^{1}$, Walter M. Weber ${ }^{2, \dagger}$, Henning Riechert ${ }^{1, \dagger}$, Steffen Münch ${ }^{3}$, \\ Ralph Rothemund ${ }^{3}$, Stephan Reitzenstein ${ }^{3}$, Alfred Forchel ${ }^{3}$, Thomas Kehagias ${ }^{4}$, Philomela Komninou ${ }^{4}$, George P. \\ Dimitrakopulos ${ }^{4}$, and Theodoros Karakostas ${ }^{4}$ \\ ${ }^{1}$ Paul-Drude-Institut für Festkörperelektronik, 5-7 Hausvogteiplatz, Berlin 10117, Germany \\ ${ }^{2}$ NaMLab gGmbH, Dresden 01187, Germany \\ ${ }^{3}$ Technische Physik, Universität Würzburg, Am Hubland, Würzburg 97074, Germany \\ ${ }^{4}$ Physics Department, Aristotle University, Thessaloniki 54124, Greece \\ ${ }^{\dagger}$ Former address: Qimonda, Munich 81730, Germany \\ Received: 5 May 2010 / Revised: 2 June 2010 / Accepted: 2 June 2010 \\ (C) The Author(s) 2010. This article is published with open access at Springerlink.com
}

\begin{abstract}
GaN nanowires have been grown by molecular beam epitaxy either catalyst-free or catalyst-induced by means of Ni seeds. Under identical growth conditions of temperature and V/III ratio, both types of GaN nanowires are of wurtzite structure elongated in the Ga-polar direction and are constricted by M-plane facets. However, the catalyst-induced nanowires contain many more basal-plane stacking faults and their photoluminescence is weaker. These differences can be explained as effects of the catalyst Ni seeds.
\end{abstract}

\section{KEYWORDS}

Nanowire, nanocolumn, molecular beam epitaxy (MBE), photoluminescence, stacking faults, catalyst

\section{Introduction}

Semiconductor nanowires (NWs) hold great promise as building blocks for novel devices in various areas like electronics, optoelectronics, photovoltaics, piezotronics, and thermoelectrics [1]. A major advantage of semiconductor NWs is that these nanoscale structures can be fabricated by fairly simple means involving selforganization effects only. Probably the most popular way is the vapor-liquid-solid (VLS) process [2], in which catalyst seeds, frequently tiny Au droplets, induce the formation of NWs. Recently, the vaporsolid-solid (VSS) process during which the catalyst remains solid was also evidenced to efficiently promote

Address correspondence to caroline.cheze@pdi-berlin.de
NW growth [3, 4]. Although widespread, the use of a catalyst raises the question whether some of the seed material is incorporated into the NWs [5-7] and adversely affects their properties, thus preventing highperformance applications. Hence, there are increasing efforts to grow NWs without any metal catalyst being used [8-13]. While significant efforts have been directed at detecting contamination by the catalyst material, so far little attention has been paid to possible differences in the quality of the products of the two approaches to fabricate NWs. For the case of GaN NWs grown by molecular beam epitaxy (MBE) with similar growth windows (V/III ratio and temperature), the formation can be either catalyst-induced 
[14] by a VSS mechanism [15] or catalyst-free [16, 17] depending on the type of substrate.

Here, we make use of this unique possibility to directly compare the structural and optical properties of NWs grown by both pathways under otherwise identical conditions in order to deduce the effect of the catalyst on the material quality. We find major differences that imply significant negative consequences if catalyst seeds are employed to synthesize semiconductor nanowires for use in devices.

\section{Experimental}

GaN NWs were grown on C-plane sapphire, $\mathrm{Si}(111)$, and on $\mathrm{Si}(001)$ substrates by plasma-assisted MBE under nitrogen-rich (N-rich) conditions. For the catalyst-induced pathway, $\mathrm{Ni}$ islands resulting from annealing of a 4 - $\AA$ thin layer were used as seeds [14]. For the catalyst-free pathway, the samples were grown on $\mathrm{Si}$ substrates cleaned with the RCA procedure. GaN was grown on each substrate for the same duration under identical conditions, i.e., a V/III ratio of 5 and a substrate temperature of $780^{\circ} \mathrm{C}$. The morphology of the NWs was investigated by scanning electron microscopy (SEM) as described in Ref. [14]. In order to assess the NW crystal structure, specimens were prepared either by NW harvesting from the substrate or by the standard mechanical thinning and ion milling processes for cross-sectional transmission electron microscopy (TEM). The optical properties of the as-grown NW samples were investigated with a photoluminescence (PL) set-up equipped with a Cryovac microscope cryostat. For excitation, we used the $325 \mathrm{~nm}$ line of a Kimmon He-Cd laser focused to a micrometer-sized spot by a $15 \times$ microscope objective. The PL signal was collected by the same objective, and analyzed by a $0.8 \mathrm{~m}$ Jobin-Yvon monochromator equipped with a cooled Si charge-coupled-device (CCD) detector.

\section{Results and discussion}

\subsection{Morphology}

All the samples were entirely covered by NWs oriented perpendicularly to the substrate as seen in the SEM images in Fig. 1. On sapphire substrates the NWs formed only in the presence of $\mathrm{Ni}$ seeds and only under N-rich conditions (Figs. 1(a) and 1(b)) [14]. In contrast, on Si substrates the NWs grew without any catalyst, but also only under N-rich conditions (Figs. 1(c)-1(f)) [17]. We therefore conclude that, as all the growth conditions were identical, whether or not a catalyst is necessary to induce the formation of NWs under the growth conditions employed depends only on the choice of substrate. The attempt to initiate the growth of catalyst-induced NWs on Si with Ni was unfruitful: Only large NiSi islands formed whereas the NWs grown on the areas free of these islands did not exhibit any catalyst at their tip. The diameters of the catalyst-induced NWs ranged from $15-60 \mathrm{~nm}$ with an average of $27 \mathrm{~nm}$ and their heights from $200-900 \mathrm{~nm}$. The NW density was $2.5 \times 10^{10} \mathrm{NWs} / \mathrm{cm}^{2}$. On Si, the diameters of the catalyst-free NWs varied from $15-100 \mathrm{~nm}$ with an average of $45 \mathrm{~nm}$ and their heights from $200-660 \mathrm{~nm}$. The majority of these NWs were shorter than the NWs induced by the Ni seeds on sapphire. Such large dispersions in diameter and length have been attributed to the coalescence of short columns and enhanced growth of thinner ones through a diffusion-induced growth mechanism [18]. The catalyst-free NW density was slightly lower, namely $1.8 \times 10^{10} \mathrm{NWs} / \mathrm{cm}^{2}$. These values are in agreement with Ref. [19]. In summary, the catalystinduced NWs are on average longer and thinner than the catalyst-free analogs.

\subsection{Crystal structure}

Using both synthetic approaches, the GaN NWs obtained were single crystalline, having the wurtzite (WZ) structure and grew along the [0001] direction, as revealed by TEM $[17,20]$ and reflection high-energy electron diffraction (RHEED) (Figs. 2(a) and 2(b)). The spots in the RHEED patterns of the catalyst-induced NWs (Fig. 2(a)) and of the catalyst-free NWs (Fig. 2(b)) correspond to electron transmission through the WZ GaN structure [17]. The reflections for the self-induced NWs are arc-shaped (Fig. 2(b)), which indicates a slight spread in tilt relative to the substrate normal [21]. For the catalyst-induced approach, the presence of Ni particles at the NW tips shows that a VLS-like 


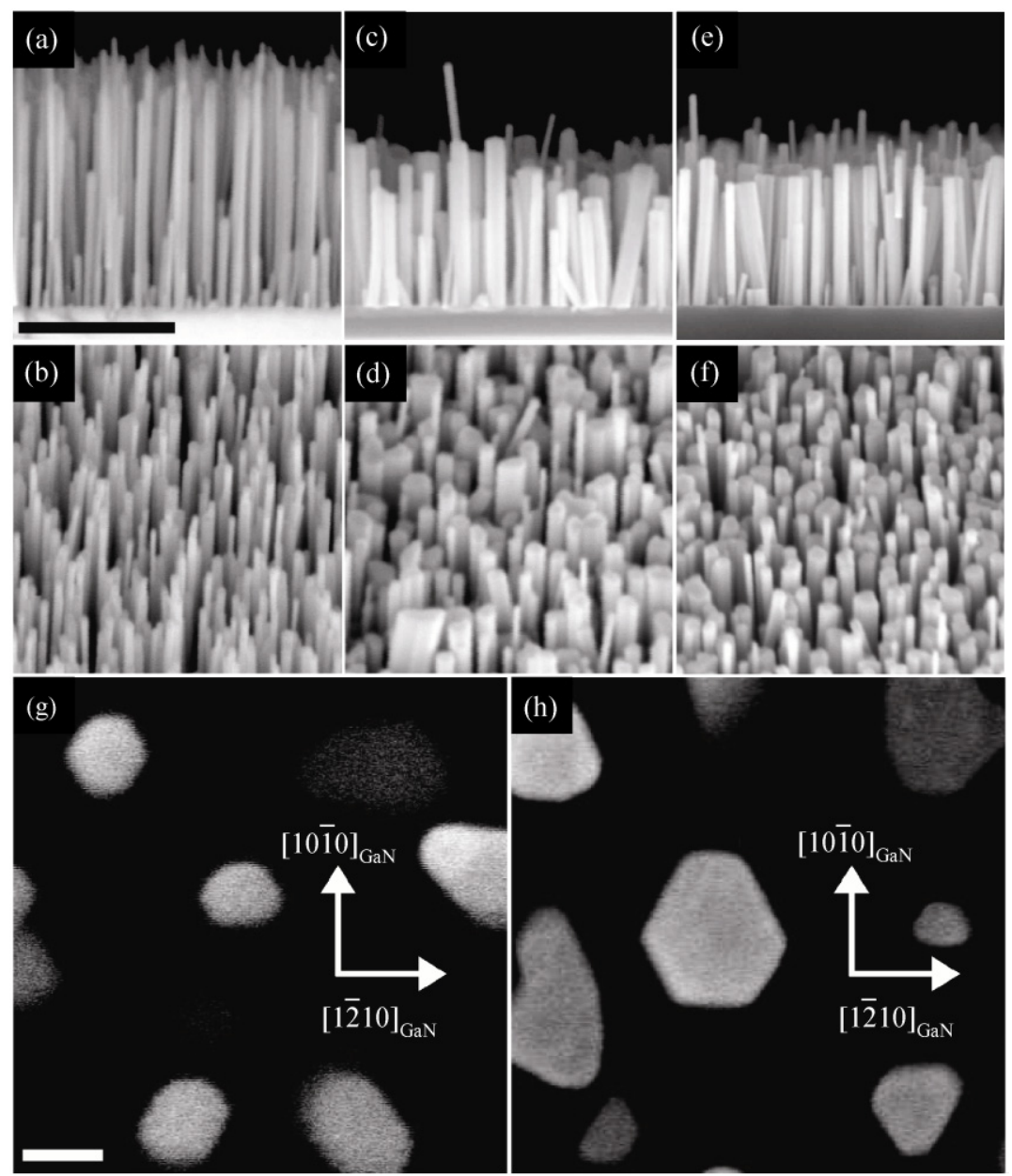

Figure 1 SEM images acquired at an angle of $90^{\circ}$ ( (top), $45^{\circ}$ (center), and $0^{\circ}$ (bottom) to the surface normal. (a), (b), (g) Niinduced NWs on C-plane sapphire. Catalyst-free-grown NWs ((c), (d), (h)) on $\mathrm{Si}(111)$ and ((e), (f)) on $\mathrm{Si}(001)$. The scale bars shown in the lower part of panel (a) and of panel (g) are $500 \mathrm{~nm}$ and $20 \mathrm{~nm}$ in length, respectively, and are valid for images ((a)-(f)) and $((\mathrm{g}),(\mathrm{h}))$, respectively. The crystallographic orientations in $(\mathrm{g})$ and $(\mathrm{h})$ refer to the lattice of GaN and are based on complementary $\mathrm{X}$-ray diffraction measurements

mechanism promotes NW growth [20], while for the catalyst-free approach the NW tips are free of any particle [17]. The facet planes which constrict the NWs were identified by SEM (Figs. 1(g) and 1(h)) and X-ray diffraction. In both approaches, M-planes constitute the NW sidewalls. The same facet orientation has been reported before for catalyst-free GaN NWs [17] but it was not clear, a priori, that catalyst-induced NWs should grow in the same shape [22-24]. Although the crystal structure of the different types of NWs is the same, their polarity could still be different. Thus, convergent beam electron diffraction (CBED) experiments were carried out (Figs. 2(c) and 2(d)). Comparison of the experimental and the corresponding simulated patterns indicated that both the catalyst-free and catalyst-induced NWs are Ga-polar ${ }^{\mathbb{D}}$. For the catalystfree grown NWs, this result is in agreement with previous reports [21, 25], but for the catalyst-assisted NWs grown on sapphire it is somewhat surprising.

(1) For the CBED measurements, the catalyst-assisted and a catalyst-free $\mathrm{NW}$ sample grown at $780{ }^{\circ} \mathrm{C}$ and at $730{ }^{\circ} \mathrm{C}$, respectively, were investigated. At the lower temperature of growth, SEM and RHEED of the catalyst-free NW sample showed no morphological difference from the high temperature sample. Thus it is legitimate to take this sample for the CBED comparison into consideration. Moreover, the results reported in Refs. [21, 26] were obtained on samples grown at $790{ }^{\circ} \mathrm{C}$, and in the range of $700-800{ }^{\circ} \mathrm{C}$, respectively. 

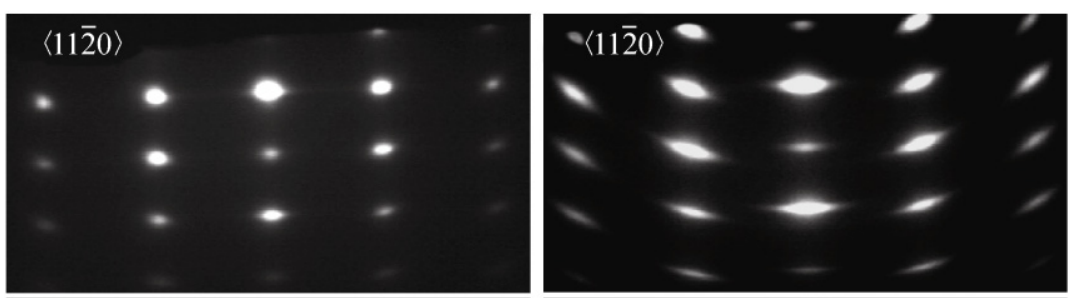

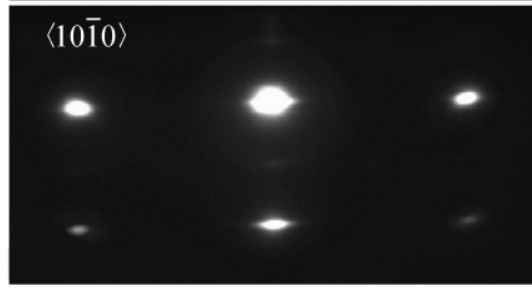

(a)
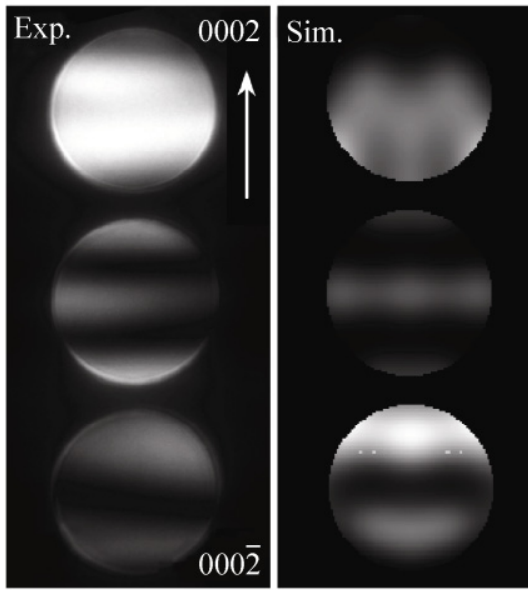

(c)

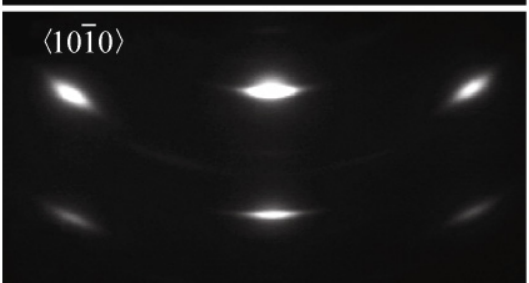

(b)

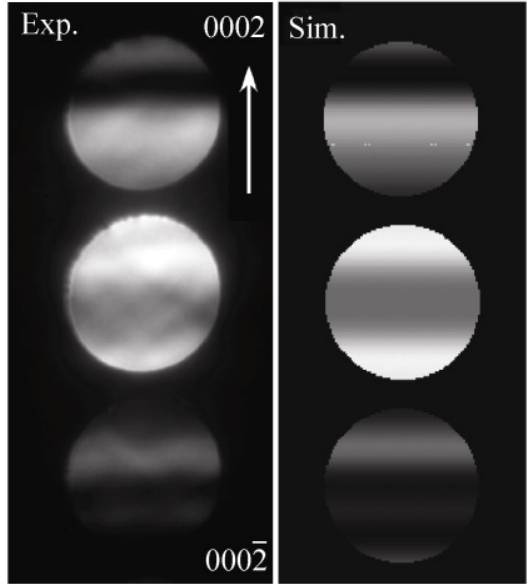

(d)

Figure 2 ((a), (b)) RHEED and ((c), (d)) CBED patterns from GaN NWs grown ((a), (c)) catalyst-induced on $\mathrm{Al}_{2} \mathrm{O}_{3}(0001)$ and ((b), (d)) catalyst-free on $\mathrm{Si}(111)$. The growth temperature of all these samples was $780{ }^{\circ} \mathrm{C}$ except for the sample investigated in (d) which was grown at $730^{\circ} \mathrm{C}$. In (a) and (b), the patterns were acquired along the $\langle 1 \overline{2} 10\rangle_{\mathrm{GaN}}$ (top) and $\langle 10 \overline{1} 0\rangle_{\mathrm{GaN}}$ (bottom) azimuths. For the CBED studies, the sample thicknesses were $4.5 \mathrm{~nm}$ and $5.3 \mathrm{~nm}$ in (c) and (d), respectively. Experimental patterns are on the left-hand side, with simulated ones on the right-hand side of each image. A Ga polarity was deduced in both cases

While we are not aware of any literature on the polarity of catalyst-induced GaN NWs, planar layers of $\mathrm{GaN}$ grown on sapphire by MBE usually exhibit N-polarity [26]. However, the mechanisms leading to that polarity of planar layers and the possible influence of the nitridation of the sapphire are rather controversial $[27,28]$. In any case, nucleation below the Ni catalyst instead of on the bare sapphire surface represents a significant change in the boundary conditions that may very well explain a difference in polarity between Ni-induced NWs and planar layers.

The defect structure of the GaN NWs was studied by TEM (Fig. 3). The catalyst-induced NWs contained many basal-plane stacking faults (SFs) randomly distributed along the NW axis. No other extended defects were found. These results are in agreement with those for similar NWs grown at lower temperature [14, 29], but the density of the SFs was higher in these cases, i.e. SF formation was reduced by increasing the growth temperature. In marked contrast, the catalyst-free NWs grown on Si were almost free of any SFs [17]. The SFs in the catalyst-induced NWs were found up to the tip, and as it will be later evidenced, both types of NWs were free of strain. Thus, the different types of substrates cannot be the reason for the discrepancy in crystal quality provided by the two approaches. In 


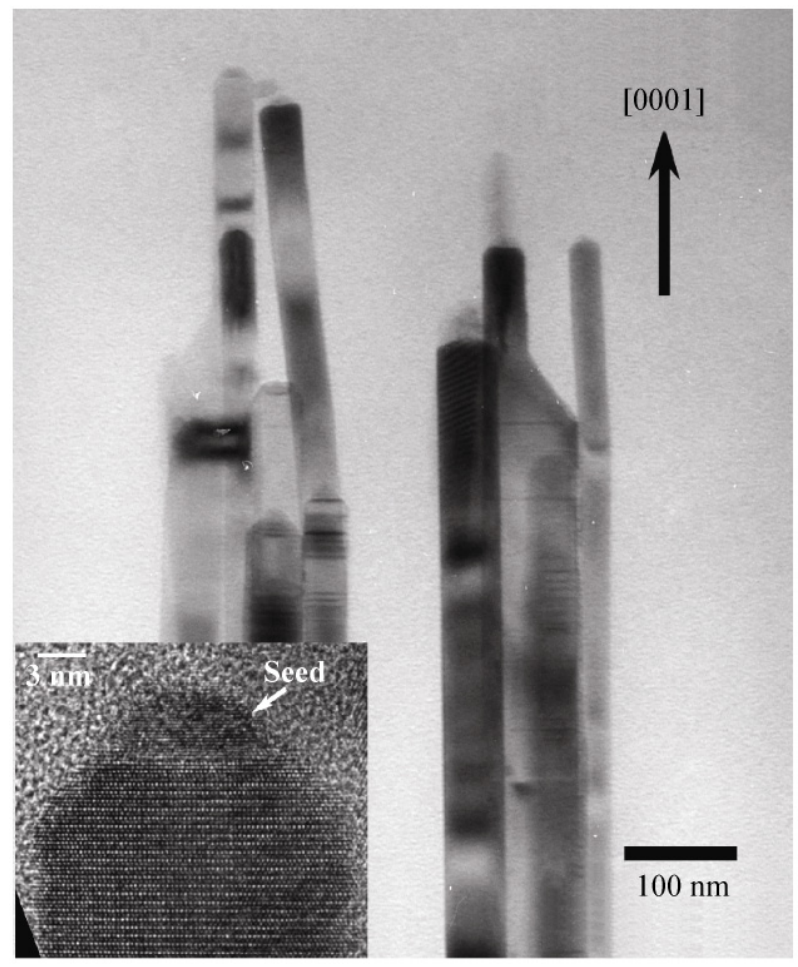

Figure 3 Cross-sectional TEM image along the $\langle 11 \overline{2} 0\rangle$ zone axis of the catalyst-induced NWs showing basal SFs within the NWs. The inset depicts a high-resolution TEM image of the tip of a NW revealing the crystalline seed particle of the catalyst

principle, the presence of many SFs in the catalystinduced NWs could be attributed to their higher vertical growth rate. However, for the following reasons this explanation can also be excluded. First, the longest catalyst-free NWs whose growth rate was close to that of the catalyst-induced NWs were free of SFs. Second, the catalyst-induced NWs grew at a vertical rate of $0.2 \mathrm{~nm} / \mathrm{s}$. This is not an unusually high growth rate for planar GaN, but the catalyst-induced NWs contain many more SFs than the latter. Instead, there are several possible explanations for the SFs which are all related to the presence of the catalyst at the NW tip where growth takes place. First, the catalyst material may affect the crystal structure of the NW material. A RHEED study [15] revealed that the solid catalyst results in a cubic NiGa structure during NW growth. Thus, the binding configuration of the GaN nucleus at the NW-catalyst interface might adopt the zinc blende (ZB) configuration because it enables a more favorable bond orientation between the Ga-top layer and the cubic GaNi. This would be similar to the use of surfactants mediating the cubic nucleation of $\mathrm{GaN}$ films [30]. In this context it would be interesting to explore whether a hexagonal catalyst has the same effect. However, the search for suitable catalysts is beyond the scope of this manuscript. Second, Ni may also be incorporated to some extent and stabilize the ZB phase. Theoretical calculations indeed showed that the incorporation of impurities, like $\mathrm{Si}, \mathrm{Mg}$, In, and C, could reduce the $\mathrm{ZB}-\mathrm{WZ}$ structural energy difference [31]. Furthermore, it has also been experimentally evidenced that Mg-doping of GaN NWs lead to the formation of stacking faults and double-twins whose density increased with the $\mathrm{Mg}$ concentration [32, 33]. Third, the catalyst introduces a triple phase line GaN-GaNi-vapor, and as described by Glas et al. for WZ GaAs NWs [34], preferential nucleation at this location could reverse whether $\mathrm{ZB}$ or $\mathrm{WZ}$ formation is favorable. Fourth, the formation of SFs could also originate from a lower surface mobility of adatoms at the NW tip, which could then be trapped at ZB sites [35-37]. This is consistent with the reduction of the SF density which was observed as the growth temperature was increased. However, the absence of SFs in the catalyst-free NWs directly identifies the catalyst particles as the origin of the reduced adatom kinetics.

\subsection{Optical properties}

For optoelectronic applications it is of crucial interest to know how the material quality affects the optical characteristics of the NWs and whether there is any difference in this respect between the two pathways. Low temperature $(10 \mathrm{~K})$ PL spectra were collected and their intensity was calibrated by comparison with that of a high quality GaN film taken as a reference sample (Fig. 4(a)). The spectra of all the samples revealed a clear emission line at $3.472 \mathrm{eV}$ related to the donor-bound exciton transition $\left(\mathrm{D}^{0}, \mathrm{X}\right)$, characteristic of strain-free GaN [38]. The sharpest peak was obtained for the NWs grown on $\mathrm{Si}(111)$ and had a full-width at half-maximum of $3.1 \mathrm{meV}$. None of the samples exhibited the yellow band, which is an indication of very low densities of the related point defects for both the catalyst-assisted and catalyst-free pathways. However, there is a strong difference in the luminescence intensity of the NWs. The overall integrated 
intensity and the peak intensity of the $\left(\mathrm{D}^{0}, \mathrm{X}\right)$ line of the NWs obtained by the catalyst-free approach are, respectively, 6 and 25 times higher than the corresponding values for the catalyst-induced NWs.

Additional emission lines were detected at $3.43 \mathrm{eV}$ for the catalyst-induced NWs, while the catalyst-free NWs exhibited emission features centered at 3.41$3.42 \mathrm{eV}$ and $3.45 \mathrm{eV}$ (Fig. 4(b)). For all these samples there appeared to be a multitude of transition lines in the range 3.41-3.43 eV. Emission in this energy range has been assigned in the bulk to structural defects, particularly SFs [39-41], and in catalyst-free NWs to structural defects at the NW-substrate interface [42]. Also in catalyst-free NWs, a blue-shift for increasing

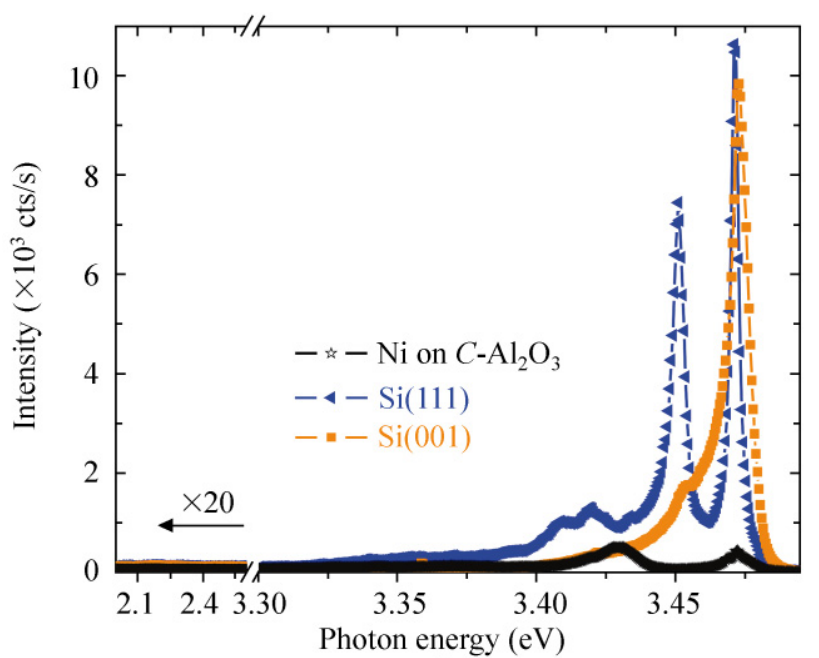

(a)

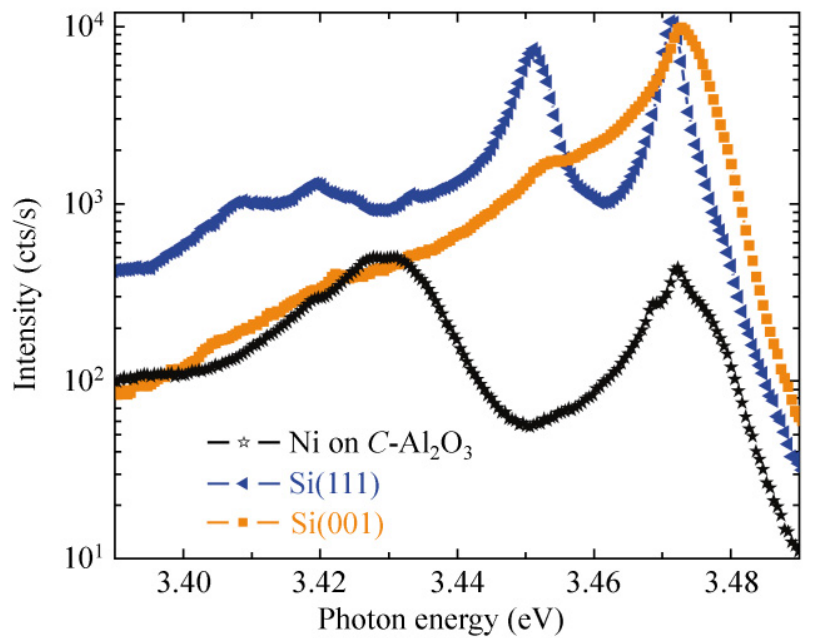

(b)

Figure 4 (a) Low temperature (10 K) PL spectra of GaN NWs grown on a sapphire substrate with $\mathrm{Ni}$ seeds, on $\mathrm{Si}(111)$, and on $\mathrm{Si}(001)$. (b) Detail of the near band edge (semi-log scale) impurity concentration $(\mathrm{Mg}$ or $\mathrm{Si}$ ) has been observed [43]. In Ni-induced GaN NWs a dominant emission at $3.437 \mathrm{eV}$ has been tentatively attributed to neutral acceptor-bound excitons related to $\mathrm{Ni}$ contamination [44]. As discussed above, the main difference in the crystal quality between the two approaches is the occurrence of SFs. Thus, a shift from $3.41-3.42 \mathrm{eV}$ for the catalyst-free NWs to $3.43 \mathrm{eV}$ for the catalystassisted NWs might be the result of SFs and/or Ni contamination. The emission of the peak in the $3.41-3.43 \mathrm{eV}$ region is much stronger compared to that of the $\left(\mathrm{D}^{0}, \mathrm{X}\right)$ transition for the catalyst-induced NWs than for the catalyst-free NWs. Also, we are not aware of any emission lines unambiguously due to $\mathrm{Ni}$ in $\mathrm{GaN}$ and SFs are known to be radiative defects. Therefore, it seems more reasonable to attribute the broad peak at $3.43 \mathrm{eV}$ to SFs.

The emission at $3.45 \mathrm{eV}$ is weaker in comparison to the $\left(\mathrm{D}^{0}, \mathrm{X}\right)$ transition in the case of growth on $\mathrm{Si}(001)$ than on $\mathrm{Si}(111)$ and not resolved in the case of growth on sapphire. This line is characteristic of catalyst-free GaN NWs grown by MBE [42]. It has been tentatively attributed to various structural features [42, 43, 45], and we cannot make any firm conclusions about the origin of this radiative transition.

The most striking of the above results is that the luminescence of the catalyst-free NWs was about 25 times stronger than that of the catalyst-induced NWs and this effect has been observed in a systematic manner for different samples. A quantitative comparison of PL intensities is difficult, but the difference between the catalyst-free and catalyst-induced NWs is so large that it must be a real effect. At this point we stress that the morphologies of the samples were very similar. The facet planes which constrict both types of NWs were identical, and the surface-to-volume ratio of both types of NWs differed by a factor of only 1.7, i.e., much less than the PL intensities. Both facts are essential to validate our comparison since surface recombination is especially critical for such nanostructures. First, the different recombination channels in these NWs do not originate from different defects characteristic of particular surfaces [24]. Second, the NW diameters are smaller than the exciton diffusion length (which may amount to $1 \mu \mathrm{m}$ at low temperature) [46]. Therefore, the NW surface-to-volume ratio does 
not significantly influence the emission intensity. Possible differences in light extraction efficiencies do not suffice as an explanation, either. As discussed above, the structural difference, i.e., the occurrence of SFs in catalyst-induced NWs, may explain additional emission lines but not the reduced PL intensity. Besides, the position of the $\left(\mathrm{D}^{0}, \mathrm{X}\right)$ peak shows that in all cases the NWs are strain-free, which indicates that the effect of the different types of substrates is limited to the base of the NWs. Thus, the results strongly suggest the presence of an additional non-radiative recombination center in the catalyst-induced NWs, and the most plausible explanation is $\mathrm{Ni}$ contamination from the catalyst-seeds. This effect has not been reported before for $\mathrm{GaN}$ doped with $\mathrm{Ni}$, which has in fact been rarely studied. However, Fe doping of GaN indeed leads to a strong decrease of the PL intensity [47] and of the luminescence decay times even at fairly low concentrations [48] by introducing very efficient non-radiative recombination channels. $\mathrm{Ni}$ and $\mathrm{Fe}$ are both $3 \mathrm{~d}$ elements, and are known to possess similar properties as dopants in conventional III- $\mathrm{V}$ semiconductors. This strongly supports the conclusion that there is unintentional incorporation of $\mathrm{Ni}$ into the catalyst-induced NWs.

Direct measurements of the $\mathrm{Ni}$ concentration in catalyst-induced NWs have been hampered by tremendous experimental challenges [5-7]. In general, it is extremely difficult to detect impurities with a concentration significantly below $1 \%$ with a spatial resolution of nanometres, as is necessary in this case. Energy-dispersive X-ray analysis of $\mathrm{Al}_{0.2} \mathrm{Ga}_{0.8} \mathrm{~N}$ catalyst-induced NWs grown via the same process and under the same conditions as the GaN NWs in this work showed $\mathrm{Ni} \mathrm{K \alpha}$ line intensities only at the background level [49]. However, the sensitivity of PL experiments to impurities is much higher than any other technique. Therefore, while we do not have direct evidence for contamination due to the catalyst seeds, our results strongly suggest it.

\section{Conclusions}

We found two main differences between catalystinduced and catalyst-free GaN NWs grown under identical conditions except for the choice of substrate.
First, the catalyst-induced NWs contained a high density of basal-plane stacking faults whereas catalyst-free growth produced material largely free of stacking faults. Second, the peaks in the PL spectra of the catalyst-free NWs were much more intense and narrower. Both of these observations can be explained as effects of the catalyst $\mathrm{Ni}$ seeds. First, Ni contamination and/or the presence of an additional phase, i.e. the catalyst seed, may be the cause of the formation of SFs. Second, the direct comparison of growth routines in the same MBE chamber under identical conditions strongly suggests that $\mathrm{Ni}$ contamination is the cause of the significantly reduced PL intensity of the catalyst-induced NWs. The use of catalyst seeds may offer an additional way to control the growth of NWs, but both the structural and optical qualities of catalyst-free nanowires are superior.

\section{Acknowledgements}

The authors are grateful to U. Jahn and A. K. Bluhm for SEM images, to B. Jenichen for XRD measurements, to L. Lari, P. R. Chalker, and M. Albrecht for discussion, and to E. Luna for a critical reading of the manuscript. This work has been supported by the EU through the IST project NODE (No. 015783) and the Marie Curie RTN PARSEM (MRTN-CT-2004-005583).

Open Access: This article is distributed under the terms of the Creative Commons Attribution Noncommercial License which permits any noncommercial use, distribution, and reproduction in any medium, provided the original author(s) and source are credited.

\section{References}

[1] Lieber, C. M.; Wang Z. L. Functional nanowires. MRS Bull. 2007, 32, 99-108.

[2] Wagner, R. S.; Ellis W. C. Vapor-liquid-solid mechanism of single crystal growth. Appl. Phys. Lett. 1964, 4, 89-90.

[3] Kamins, T. I.; Williams, R. S.; Basile, D. P.; Hesjedal, T.; Harris, J. S. Ti-catalyzed Si nanowires by chemical vapor deposition: Microscopy and growth mechanisms. J. Appl. Phys. 2001, 89, 1008-1016.

[4] Persson, A. I.; Larsson, M. W.; Stenstrom, S.; Ohlsson, B. J.; Samuelson, L.; Wallenberg, L. R. Solid-phase diffusion 
mechanism for GaAs nanowire growth. Nat. Mater. 2004, 3 , 677-681.

[5] Putnam, M. C.; Filler, M. A.; Kayes, B. M.; Kelzenberg, M. D.; Guan, Y.; Lewis, N. S.; Eiler, J. M.; Atwater, H. A. Secondary ion mass spectrometry of vapor-liquid-solid grown, Au-catalyzed, Si wires. Nano Lett. 2008, 8, 3109-3113.

[6] Oh, S. H.; Benthem, K. V.; Molina, S. I.; Borisevich, A. Y.; Luo, W.; Werner, P.; Zakharov, N. D.; Kumar, D.; Pantelides, S. T.; Pennycook, S. J. Point defect configurations of supersaturated $\mathrm{Au}$ atoms inside Si nanowires. Nano Lett. 2008, 8, 1016-1019.

[7] Allen, J. E.; Hemesath, E. R.; Perea, D. E.; Lensch-Falk, J. L.; Li, Z. Y.; Yin, F.; Gass, M. H.; Wang, P.; Bleloch, A. L.; Palmer, R. E.; Lauhon, L. J. High-resolution detection of $\mathrm{Au}$ catalyst atoms in Si nanowires. Nat. Nanotechnol. 2008, 3, 168-173.

[8] Zhang, R. Q.; Lifshitz, Y.; Lee, S. T. Oxide-assisted growth of semiconducting nanowires. Adv. Mater. 2003, 15, 635-640.

[9] Noborisaka, J; Motohisa, J; Fukui, T. Catalyst-free growth of GaAs nanowires by selective-area metalorganic vapourphase epitaxy. Appl. Phys. Lett. 2005, 86, 213102.

[10] Mohammad, S. N. Self-catalysis: A contamination-free, substrate-free growth mechanism for single-crystal nanowire and nanotube growth by chemical vapor deposition. $J$. Chem. Phys. 2006, 125, 094705.

[11] Mandl, B.; Stangl, J.; Mårtensson, T.; Mikkelsen, A.; Eriksson, J.; Karlsson, L. S.; Bauer, G.; Samuelson, L.; Seifert, W. Au-free epitaxial growth of InAs nanowires. Nano Lett. 2006, 6, 1817-1821.

[12] Kim, B. S.; Koo, T. W.; Lee, J. H.; Kim, D. S.; Jung, Y. C.; Hwang, S. W.; Choi, B. L.; Lee, E. K.; Kim, J. M.; Whang, D. Catalyst-free growth of single-crystal silicon and germanium nanowires. Nano Lett. 2009, 9, 864-869.

[13] Kuykendall, T.; Ulrich, P.; Aloni, S.; Yang, P. Complete composition tunability of InGaN nanowires using a combinatorial approach. Nat. Mater. 2007, 6, 951-956.

[14] Geelhaar, L.; Chèze, C.; Weber, W. M.; Averbeck, R.; Riechert, H.; Kehagias, T.; Komninou, P.; Dimitrakopulos, G. P.; Karakostas, T. Axial and radial growth of Ni-induced GaN nanowires. Appl. Phys. Lett. 2007, 91, 093113.

[15] Chèze, C. Investigation and comparison of GaN nanowire nucleation and growth by the catalyst-assisted and selfinduced approach. Ph.D. Dissertation, Humboldt-Universität zu Berlin, Germany, 2010.

[16] Yoshizawa, M.; Kikuchi, A.; Mori, M.; Fujita, N.; Kishino, K. Growth of self-organized $\mathrm{GaN}$ nanostructures on $\mathrm{Al}_{2} \mathrm{O}_{3}(0001)$ by RF-radical source molecular beam epitaxy. Jpn. J. Appl. Phys. 1997, 36, L459-L462.

[17] Calleja, E.; Ristić, J.; Fernández-Garrido, S.; Cerutti, L.; Sánchez-García, M. A.; Grandal, J.; Trampert, A.; Jahn, U.; Sánchez, G.; Griol, A.; Sánchez, B. Growth, morphology, and structural properties of group-III-nitride nanocolumns and nanodisks. Phys. Status Solidi B 2007, 244, 2816-3837.

[18] Debnath, R. K.; Meijers, R.; Richter, T.; Stoica, T.; Calarco, R.; Lüth, H. Mechanism of molecular beam epitaxy growth of GaN nanowires on Si(111). Appl. Phys. Lett. 2007, 90, 123117.

[19] Calarco, R.; Meijers, R. J.; Debnath, R. K.; Stoica, T.; Sutter, E.; Lüth, H. Nucleation and growth of GaN nanowires on $\mathrm{Si}(111)$ performed by molecular beam epitaxy. Nano Lett. 2007, 7, 2248-2251.

[20] Kehagias, T.; Komninou, P.; Dimitrakopulos, G. P.; Chèze, C.; Geelhaar, L.; Riechert, H.; Karakostas, T. Atomic-scale configuration of catalyst particles on GaN nanowires. Phys. Status Solidi C 2008, 5, 3716-3719.

[21] Furtmayr, F.; Vielemeyer, M.; Stutzmann, M.; Arbiol, J.; Estradé, S.; Peirò, F.; Morante, J. R.; Eickhoff, M. Nucleation and growth of $\mathrm{GaN}$ nanorods on $\mathrm{Si}(111)$ surfaces by plasma-assisted molecular beam epitaxy-The influence of Si- and Mg-doping. J. Appl. Phys. 2008, 104, 034309.

[22] Qian, F.; Li, Y.; Gradečak, S.; Park, H. G.; Dong, Y.; Ding, Y.; Wang, Z. L.; Lieber, C. M. Multi-quantum-well nanowire heterostructures for wavelength-controlled lasers. Nat. Mater. 2008, 7, 701-706.

[23] Kuykendall, T.; Pauzaukie, P. J.; Zhang, Y. F.; Goldberger, J.; Sirbuly, D.; Denlinger, J.; Yang, P. D. Crystallographic alignment of high-density gallium nitride nanowire arrays. Nat. Mater. 2004, 3, 524-528.

[24] Chin, A. H.; Ahn, T. S.; Li. H; Vaddiraju. S; Bardeen, C. J.; Ning, C. Z.; Sunkara, M. K. Photoluminescence of GaN nanowires of different crystallographic orientations. Nano Lett. 2007, 7, 626-631.

[25] Cherns, D.; Meshi, L.; Griffiths, I.; Khongphetsak, S.; Novikov, S. V.; Farley, N.; Campion, R. P.; Foxon, C. T. Defect reduction in $\mathrm{GaN} /(0001)$ sapphire films grown by molecular beam epitaxy using nanocolumn intermediate layers. Appl. Phys. Lett. 2008, 92, 121902.

[26] Smith, A. R.; Feenstra, R. M.; Greve, D. W.; Neugebauer, J.; Northrup, J. E. Reconstructions of the GaN(0001) surface. Phys. Rev. Lett. 1997, 79, 3934-3937.

[27] Yoshikawa, A.; Xu, K. Polarity selection process and polarity manipulation of GaN in MOVPE and RF-MBE growth. Thin Solid Films 2002, 412, 38-43. 
[28] Georgakilas, A.; Mikroulis, S.; Cimalla, V.; Zervos, M.; Kostopoulos, A.; Komninou, P.; Kehagias, T.; Karakostas, T. Effects of the sapphire nitridation on the polarity and structural properties of GaN layers grown by plasma-assisted MBE. Phys. Status Solidi A 2001, 188, 567-570.

[29] Lari, L.; Murray, R. T.; Bullough, T. J.; Chalker, P. R.; Gass, M.; Chèze, C.; Geelhaar, L.; Riechert, H. Defect characterization and analysis of III $-\mathrm{V}$ nanowires grown by Ni-promoted MBE. Phys. Status Solidi A 2008, 205, 2589-2592.

[30] Zhao, Y.; Tu, W.; Bae, I. T.; Seong, T. Y. Growth of cubic GaN by phosphorus-mediated molecular beam epitaxy. Appl. Phys. Lett. 1999, 74, 3182-3184.

[31] Chisholm, J. A.; Bristowe, P. D. Ab initio study of the effect of doping on stacking faults in GaN. J. Cryst. Growth 2001, 230, 432-437.

[32] Cimpoiasu, E.; Stern, E.; Klie, R.; Munden, R. A.; Cheng, G.; Reed, M. A. The effect of Mg doping on GaN nanowires. Nanotechnology 2006, 17, 5735-5739.

[33] Arbiol, J.; Estradé, S.; Prades, J. D.; Cirera, A.; Furtmayr, F.; Stark, C.; Laufer, A.; Stutzmann, M.; Eickhoff, M.; Gass, M. H., et al. Triple-twin domains in $\mathrm{Mg}$ doped $\mathrm{GaN}$ wurtzite nanowires: Structural and electronic properties of this zincblende-like stacking. Nanotechnology 2009, 20, 145704.

[34] Glas, F.; Harmand, J. C.; Patriarche, G. Why does wurtzite form in nanowires of III $-\mathrm{V}$ zinc-blende semiconductors? Phys. Rev. Lett. 2007, 99, 146101.

[35] Tarsa, E. J.; Heying, B.; Wu, X. H.; Fini, P.; DenBaars, S. P.; Speck, J. S. Homoepitaxial growth of GaN under Ga-stable and N-stable conditions by plasma-assisted molecular beam epitaxy. J. Appl. Phys. 1997, 82, 5472-5479.

[36] Zywietz, T.; Neugebauer, J.; Scheffler, M. Adatom diffusion at $\mathrm{GaN}(0001)$ and $(000 \overline{1})$ surfaces. Appl. Phys. Lett. 1998, 73, 487-489.

[37] Shi, B. M.; Xie, M. H.; Wu, H. S.; Wang, N.; Tong, S. Y. Transition between wurtzite and zinc-blende GaN: An effect of deposition condition of molecular-beam epitaxy. App. Phys. Lett. 2006, 89, 151921.

[38] Robins, L. H., Bertness, K. A., Barker, J. M., Sanford, N. A.; Schlager, J. B. Optical and structural study of $\mathrm{GaN}$ nanowires grown by catalyst-free molecular beam epitaxy. I. Nearband-edge luminescence and strain effects. J. Appl. Phys. 2007, 101, 113505 and references therein.

[39] Liu, R.; Bell, A.; Ponce, F. A.; Chen, C. Q.; Yang, J. W.; Kahn, M. A. Luminescence from stacking faults in gallium nitride. Appl. Phys. Lett. 2005, 86, 021908.

[40] Paskov, P. P.; Schifano, R.; Monemar, B.; Paskova, T.;
Figge, S.; Hommel, D. Emission properties of $a$-plane GaN grown by metal-organic chemical-vapor deposition. J. Appl. Phys. 2005, 98, 093519.

[41] Salviati, G.; Albrecht, M.; Zanotti-Fregonara, C.; Armani, N.; Mayer, M.; Shreter, Y.; Guzzi, M.; Melnik, Y.; Vvassilevski, K.; Dmitriev, V. A.; Strunk, H. P. Cathodoluminescence and transmission electron microscopy study of the influence of crystal defects on optical transitions in GaN. Phys. Status Solidi A 1999, 171, 325-339.

[42] Calleja, E. ; Sánchez-García, M. A.; Sánchez, F. J.; Calle, F.; Naranjo, F. B.; Muñoz, E.; Jahn, U.; Ploog, K. Luminescence properties and defects in GaN nanocolumns grown by molecular beam epitaxy. Phys. Rev. B 2000, 62, 16826-16834.

[43] Furtmayr, F.; Vielemeyer, M.; Stutzmann, M.; Laufer, A.; Meyer, B. K.; Eickhoff, M. Optical properties of Si- and $\mathrm{Mg}$-doped gallium nitride nanowires grown by plasmaassisted molecular beam epitaxy. J. Appl. Phys. 2008, 104, 074309.

[44] Yoo, J.; Hong, Y. J.; An, S. J.; Yi, G. C.; Chon, B.; Joo, T.; Kim, J. W.; Lee, J. S. Photoluminescent characteristics of Nicatalyzed GaN nanowires. Appl. Phys. Lett. 2006, 89, 043124.

[45] Corfdir, P.; Lefebvre, P.; Ristić, J.; Valvin, P.; Calleja, E.; Trampert, A.; Ganière, J. D.; Deveaud-Plédran, B. Timeresolved spectroscopy on $\mathrm{GaN}$ nanocolumns grown by plasma assisted molecular beam epitaxy on Si substrates. J. Appl. Phys. 2009, 105, 013113.

[46] Brandt, O. ; Yang, B. ; Wünsche, H. J. ; Jahn, U. ; Ringling, J.; Paris, G.; Grahn, H. T.; Ploog, K. H. Impact of exciton diffusion on the optical properties of thin GaN layers. Phys. Rev. B 1998, 58, R13407-R13410.

[47] Azize, M.; Leroux, M.; Laugt, M.; Gibart, P.; Bougrioua, Z. Strain and microstructure in Fe-doped GaN layers grown by low pressure metalorganic vapour phase epitaxy. Phys. Status Solidi A 2006, 203, 1744-1748.

[48] Aggerstam, T.; Pinos, A.; Marcinkevicius, S.; Linnarsson, M.; Lourdudoss, S. Electron and hole capture cross-sections of Fe acceptors in GaN:Fe epitaxially grown on sapphire. $J$. Electron. Mater. 2007, 36, 1621-1624.

[49] Lari, L.; Murray, R. T.; Bullough, T. J.; Chalker, P. R.; Gass, M. H.; Chèze, C.; Geelhaar, L.; Riechert, H. Electron microscopy analysis of $\mathrm{AlGaN} / \mathrm{GaN}$ nanowires grown by catalyst-assisted molecular beam epitaxy. In Microscopy of Semiconducting Materials 2007: Proceedings of the 15th Conference, 2-5 April 2007, Cambridge, UK; Cullis A. G; Midgley, P. A., Eds; Springer \& Canopus Publishing Limited 2007, pp. 221-224. 